PROCEEDINGS OF THE

AMERICAN MATHEMATICAL SOCIETY

Volume 45, Number 2, August, 1974

\title{
GLOBAL GEOMETRY OF POLYGONS. I: THE THEOREM OF FABRICIUS-BJERRE
}

\author{
THOMAS F. BANCHOFF
}

ABSTRACT. Deformation methods provide a direct proof of a polygonal analogue of a theorem proved by $\mathrm{Fabricius-Bjerre} \mathrm{and} \mathrm{by} \mathrm{Halpern} \mathrm{relating}$ the numbers of crossings, pairs of inflections, and lines of double tangency for smooth closed plane curves.

Let $X:[a, b] \rightarrow E^{2}$ describe a closed curve with a finite number $C$ of crossings, a finite number $F$ of inflection points (or inflection intervals), and a finite number of double support lines (i.e. lines containing two points of the curve each with a neighborhood lying to one side of the line, $\mathrm{I}_{t}$ being the number for which the neighborhoods lie on opposite sides of the line and $\mathrm{II}_{t}$ being the number with both neighborhoods to the same side). For a convex curve all these numbers are zero. Examination of a few examples leads to the conjecture that $C+1 / 2 F+\mathrm{I}_{t}-\mathrm{II}_{t}=0$, and in [2] Halpern announced a proof of this result for smooth curves satisfying certain regularity conditions. The proof described there uses techniques of critical points for vector fields and winding numbers and requires that the curve be of differentiability class $C^{4}$. The result was discovered by Fabricius-Bjerre [3], but again the proof made use of regularity slightly stronger than $C^{1}$.

In this note we present an elementary proof of this result for polygonal curves in the plane. As in [2], this result generalizes to the case of mappings of an arbitrary 1-manifold into the plane (Theorem $1^{\prime}$ ).

The techniques developed in this paper will be used in subsequent investigations of polygonal analogues of a number of other global theorems on plane and space curves. There is also a sense in which these techniques fit in with the polyhedral analogue of catastrophe theory as developed in [1].

Definition. By a polygon we mean a mapping $X:[a, b] \rightarrow E^{2}$ of a closed interval into the Euclidean plane such that for some finite subdivision, $a=$ $t_{0}<t_{1}<\cdots<t_{m}=b$, the mapping $X$ restricted to the interval $t_{i} \leq t \leq t_{i+1}$

Presented to the Society, April 27, 1973 under the title Polygonal methods in global curve theory; received by the editors January 17, 1973 and, in revised form, August 29, 1973.

AMS (MOS) subject classifications (1970). Primary 53A05, 57C35; Secondary 55A25.

Key words and phrases. Polygon, inflections, double tangency, support line, deformations. 
is linear, so in this interval

$$
X(t)=\frac{t_{i+1}-t}{t_{i+1}-t_{i}} X\left(t_{i}\right)+\frac{t-t_{i}}{t_{i+1}-t_{i}} X\left(t_{i+1}\right) .
$$

The points $X\left(t_{i}\right)$ are abbreviated $X_{i}$ and are called the vertices of the polygon. If $X_{0}=X_{m}$, then $X$ is a closed m-gon and in this case we consider all indices to be reduced modulo $m$.

A polygon is said to be general if the vertices $\left\{X_{i} \mid i=0, \cdots, m-1\right\}$ are in general position, so no three vertices are collinear.

For a general polygon $X$, we may make precise the four indices referred to in the introduction: Let $C(X)$ be the number of crossings, i.e. pairs of indices $(i, j)$ with $0 \leq i<j \leq m-1$ such that the edge $\left[X_{i}, X_{i+1}\right]$ meets the edge $\left[X_{j}, X_{j+1}\right]$. Let $F(X)$ be the number of inflection edges, i.e. edges $\left[X_{i}, X_{i+1}\right]$ such that $X_{i+1}$ lies on one side of the line containing the edge and $X_{i+2}$ lies on the other. By a double support line we mean a pair of indices $(i, j)$ with $0 \leq i<j \leq m-1$ such that $X_{i-1}$ and $X_{i+1}$ lie on the same side of the line through $X_{i}$ and $X_{j}$ and so do $X_{j-1}$ and $X_{j+1}$. Let $I_{t}(X)$ denote the number of double support lines with $X_{i-1}$ and $X_{j+1}$ on opposite sides of the line through $X_{i}$ and $X_{j}$ and let $\operatorname{II}_{t}(X)$ denote the number with $X_{i-1}$ and $X_{j+1}$ on the same side. We define the function

$$
H(X)=C(X)+1 / 2 F(X)+I_{t}(X)-\mathrm{II}_{t}(X) .
$$

We may now state the main result:

Theorem 1. If $X$ is general, then $H(X)=0$.

This theorem is clearly true for a convex polygon, for which all indices are zero. We shall prove the theorem by deforming the given polygon $X$ in a certain way into a convex $m$-gon and by showing that the combination of indices remains unchanged throughout the deformation.

Definition. By an elementary deformation of a general polygon $X$ determined by a cycle of vertices $\left\{X_{i} \mid i=0, \cdots, m-1\right\}$ we mean a 1 -parameter family $X(u)=\left\{X_{i}(u) \mid i=0, \cdots, m-1\right\}, u_{k} \leq u \leq u_{k+1}$, of polygons such that for some index $j$ and some point $X_{j}^{\prime}, X_{i}(u)=X_{i}$ for all $u$ if $i \neq j$ and

$$
X_{j}(u)=\left(\frac{u_{k+1}-u}{u_{k+1}-u_{k}}\right) X_{j}+\left(\frac{u-u_{k}}{u_{k+1}-u_{k}}\right) X_{j}^{\prime}
$$

This elementary deformation is said to be in general position if $X(u)$ is general for all but a finite number of the parameter values $u_{k} \leq u \leq u_{k+1}$, and if for these exceptional values, there is exactly one triple of collinear vertices.

Lemma 1. For almost all choices of the vertex $X_{j}^{\prime}$ the elementary defor- 
mation described above is in general position.

Proof. Consider the finitely many lines containing distinct vertex pairs of the general polygon $X$. The only way that the elementary deformation can fail to be in general position is if a line containing $X_{j}$ and $X_{j}^{\prime}$ contains a point of intersection of two lines from this collection, so we get an elementary deformation in general position by choosing $X_{j}^{\prime}$ in the complement of the finite set of lines from $X_{j}$ to these intersection points.

By a piecewise elementary deformation of a general polygon $X$ we mean a 1-parameter family $X(u)$ of polygons such that for some finite subdivision $u_{0}<u_{1}<\cdots<u_{r}$ of the parameter space, $X\left(u_{0}\right)=X$ and each family $X(u)$, $u_{k} \leq u \leq u_{k+1}$, is an elementary deformation. Such a deformation is said to be in general position if each $X\left(u_{k}\right)$ is general and if each elementary deformation is in general position.

Lemma 2. If $X$ is general then there is a piecewise elementary deformation $X(u), u_{0} \leq u \leq u_{r}$, in general position such that $X\left(u_{r}\right)$ is a convex m-gon.

Proof. Begin with a regular $m$-gon $Y$ and proceed inductively to move the vertices $X_{j}$ of $X$ to vertices $X_{j}^{\prime}$ near the vertices $Y_{j}$ of $Y$ so that each successive elementary deformation is in general position and so that at each stage the polygon with vertices $X_{0}^{\prime}, X_{1}^{\prime}, \ldots, X_{j}^{\prime}, Y_{j+1}, \ldots, Y_{m-1}$ is convex.

We now need only show that for an elementary deformation in general position, the changes $\Delta C, \Delta F, \Delta \mathrm{I}_{i}$, and $-\Delta \mathrm{II}_{t}$ add up to 0 as we pass any collineation. This we show simply by exhibiting these differences explicitly for the basic collineations which can occur, and by observing that for each of these

$$
\Delta H=\Delta C+\Delta(1 / 2) F+\Delta \mathrm{I}_{t}-\Delta \mathrm{II}_{t}=0
$$

We consider five different types of collineations:

(a) If no two of the three collinear vertices lie in an edge of $X$, then none of the indices involving crossings or inflections are affected at all and double tangency indices stay the same.

(b) If precisely two of the three vertices lie in an edge of $X$ but the adjacent vertices of the third vertex are on opposite sides of the collineation line, then if the vertex does not pass through the edge we have no change of indices, and if it does, then one crossing edge is lost and one is gained so the change in each index is zero.

(c) If precisely two of the three vertices lie on an edge and the adjacent vertices of the third vertex are on the same side of the collineation line, then 
we have eight possibilities, which we list schematically, together with the changes in the four indices, as cases 1 through 8 in the table.

(d) If the three vertices are consecutive but the polygon is not a triangle, then we have four possibilities, cases 9 through 12 in the table.

(e) If the polygon is a triangle, then all indices are zero before and after the deformation.

If $X$ is a mapping of a compact 1 -manifold (not necessarily connected) into the plane, then the definition in the connected case may be extended to yield indices $C(X), F(X), \mathrm{I}_{t}(X)$, and $\mathrm{II}_{t}(X)$. A polygonal mapping $X$ of a 1 -manifold into the plane corresponds to a finite collection of $m_{l}$-gons $X^{l}$, $l=0, \cdots, n-1$, and $X$ is said to be general if the collection of all vertices of all of the polygons $X^{l}$ is in general position.

BEFORE

1.

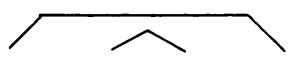

2.

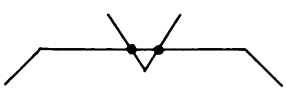

3.

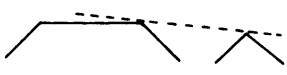

4.

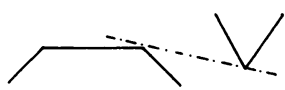

5.

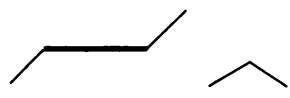

6.

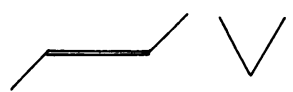

7.

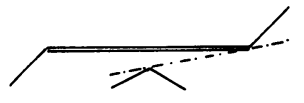

8.

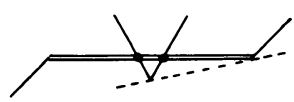

9.

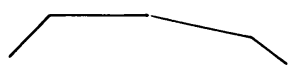

10.

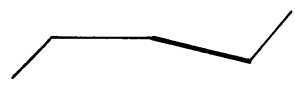

11.

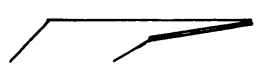

12.

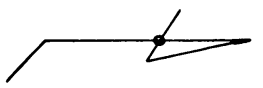

AFTER
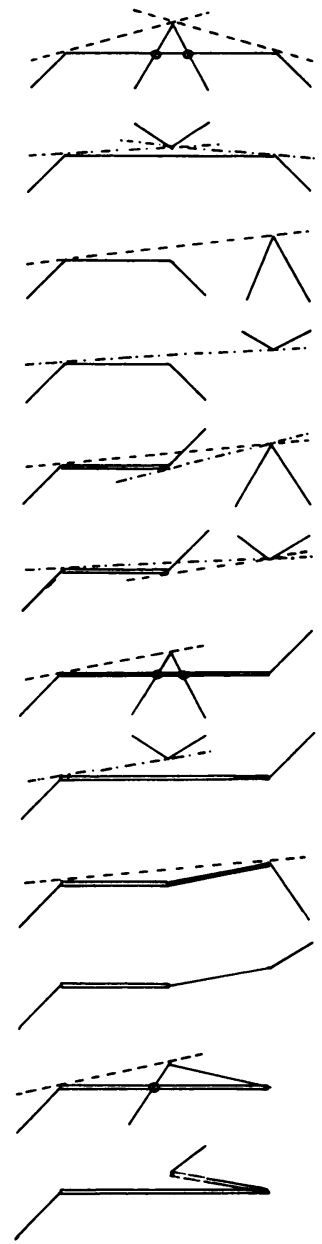

$\Delta C \quad 1 / 2 \Delta F \Delta \mathrm{I}_{t} \Delta \mathrm{II}_{t}$

2002

$\begin{array}{llll}-2 & 0 & 2 & 0\end{array}$

$\begin{array}{llll}0 & 0 & 0 & 0\end{array}$

$\begin{array}{llll}0 & 0 & 0 & 0\end{array}$

$\begin{array}{llll}0 & 0 & 1 & 1\end{array}$

$\begin{array}{llll}0 & 0 & 1\end{array}$

$\begin{array}{llll}2 & 0 & -1 & 1\end{array}$

$\begin{array}{llll}-2 & 0 & 1 & -1\end{array}$

$\begin{array}{llll}0 & 1 & 0 & 1\end{array}$

0000

1001

$\begin{array}{llll}-1 & 1 & 0 & 0\end{array}$ 
Theorem $\mathbf{l}^{\prime}$. As before, for a general polygonal mapping of a compact 1-manifold $X, H(X)=C(X)+1 / 2 F(X)+\mathrm{I}_{t}(X)-\mathrm{II}_{t}(X)=0$.

Proof. Let $Y$ be a nested collection of disjoint convex $m_{l}$-gons $Y^{l}$, $l=0,1, \cdots, n-1$, such that each polygon $Y^{l}$ is contained in the open region bounded by $Y^{l+1}$, and such that the collection of all vertices of polygons in $Y$ is in general position. Then $C(Y)=0=F(Y)=\mathrm{I}_{t}(Y)=\mathrm{II}_{t}(Y)$ so $H(Y)=0$, and the theorem follows as in the case of Theorem 1 by finding a piecewise elementary deformation in general position from $X$ to be a general polygonal mapping $X^{\prime}$ with vertices near those of $Y$.

\section{BIBLIOGRAPHY}

1. T. Banchoff, Polyhedral catastrophe the ory. I: Maps of the line to the line, Dynamical Systems, Academic Press, New York and London, 1973.

2. B. Halpern, Global theorems for closed plane curves, Bull. Amer. Math. Soc. 76 (1970), 96-100. MR 41 \#7541.

3. Fr. Fabricius-Bjerre, On the double tangents of plane closed curves, Math. Scand. 11 (1962), 113-116. MR 28 \#4439.

DEPARTMENT OF MATHEMATICS, BROWN UNIVERSITY, PROVIDENCE, RHODE ISLAND 02912. 